NER2 004382

ULTRAHIGH PRESSURE LASER-DRIVEN SHOCK WAVE EXPERIMENTS

R. J. Trainor, N. C. Holmes, R. A. Anderson University of California, Lawrence Livermore National Laboratory Livermore, California 94550

This paper was prepared for submittal to Proceedings of tine American Physical Society Topical Conference on Shockwaves in Condensed Matter Menlo Park, California

June 22 through 24,1981

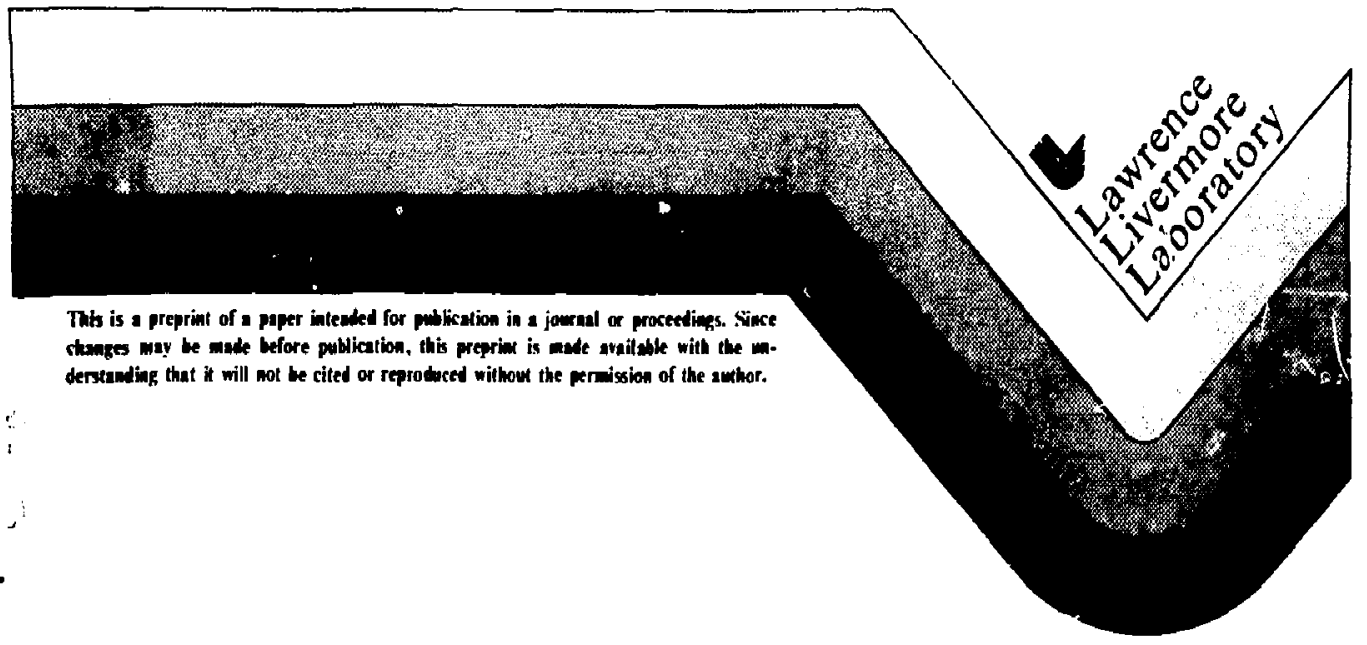




\section{ULTRAHIGH PRESSURE LASER-DRIVEN SHOCK WAVE EXPERIMENTS*}

R. J. Trainor, N. C. Holmes, R. A. Anderson

University of Califoraia, Lawrence Livermore National laboratory Livermore, California 94550

\section{ABSTRACT}

We review recent laser-driven shock wave experiments, with a view toward assessing the prospects of making accurate physical properties measurements at ultrahigh pressures. Recent experimental results on the scaling of shock pressure with laser intensity and wavelength are presented, and preliminary impedance matching experiments are discussed.

\section{Introduction}

We have known for many years that very high pressure shock waves can be produced by intense laser pulses, even though many of the properties of such strong shocks have only been characterized very recently, and some remain poorly understood. A more challenging issue is whether or not it is possible to make accurate and meaningful physical property measurements under the conditions of a strong laser-generated shock compression. To evaluate the prospects of doing this, we need the answers to several key questions. How high are the achievable shock pressures? What are the effects of preheating by suprathermal electrons from the laser plasma and of shock attenuation due to the short lifetime of the laser pulse? How do we deal with them? What are the constraints on beam uniformity? Can we fabricate and characterize targets with the necessary submicron tolerances? What kinds of experiments are possible with present diagnostic capabilities? In this paper we use the results of recent experiments at LLNL as guidance in exploring these questions and speculating about some future directions in lasergenerated shock wave research.

\section{Strength of Laser Generated Shocks}

Simple analytic models developed many years ago(1) predict that the maximum shock pressure $P_{s}$ is an increasing function of incident laser intensity $I_{0}$, varying as $I_{0}^{\alpha}$. This result is substantiated by more detailed calculations (2) using laser-plasmahydrodynamics codes such as LASNEX. (3) Theoretically, we expect $\alpha=0.6-0.8$. These predictions have recently been confirmed experimentally for laser intensities between $10^{12}$ and about $5 \times 10^{14} \mathrm{w} / \mathrm{cm}^{2}$, as shown in Figure 1 . Here we have plotted results

*hork performed under the auspices of the U.S. Department of Energy by Lawrence Livermore National Laboratory under contract \#W-7405-Eng-48. 
for aluminum obtained by Grun et al., (4) which extend up to about $10^{14} \mathrm{w} / \mathrm{cm}^{2}$. The solid line is a best fit to the shock pressures they inferred from ion time-of-flight and ballistic pendulum measurements and corresponds to $\alpha=0.8$. These are timeintegrated measurements and thus probably give a good measure of the peak pressure. We have measured shock velocities produced by 300 ps Gaussian pulses in stepped aluminum targets, using an ultrafast imaging system $(5)$ to record shock transit times across premeasured steps. The results are used to infer the pressure $20 \mathrm{~km}$ deep inside the target, where it has been hydrodynamically attenuated somewhat; hence the lower pressures than would be predicied by extrapolation of Grun's measurements. We find $\alpha=0.65$. Both sets of data are in good agreement with LASNEX simulations.

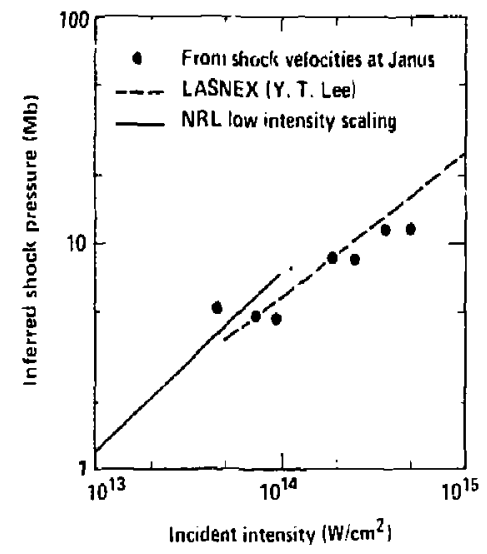

Figure 1. Inferred shock pressure vs incident intensity. The solid line, corresponding to $\alpha=0.8$, is a fit to the data of Grun et al. The points, obtained from shock velocity measurements, correspond to $\alpha$ $=0.65$.

At higher intensities, there is some evidence that the powerlaw scaling may break down and that the peak pressure may actually saturate as intensity is increased above $210^{15} \mathrm{~W} / \mathrm{cm}^{2} .(6)$ To test the limits of high pressure achievable in a preheat-free, planar laser-driven shock experiment, we have measured the velocity of a strong shock produced in a gold target by ten arms of the Shiva laser. The target consisted of a 22- $\mu \mathrm{m}$-thick layer of aluminum

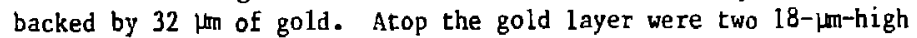
gold steps, one on each side of a 150-ןm-wide channel. The roughness of the gold surface was less than $0.3 \mathrm{\mu m}$, and the step height was measured to better than $0.2 \mathrm{~lm}$ accuracy. The aluminum layer was used to exploit the shock impedance mismatch between aluminum and gold to produce a stronger shock. The thick gold layer was required to assure a preheat-free shock.

The target was irradiated on the aluminum side with a 625 ps FWHM pulse of incident intensity $2.9 \times 10^{15} \mathrm{~W} / \mathrm{cm}^{2}$. The beams were overlapped to produce a spatially uniform spot with intensity variations estimated to be no greater than about 15 percent. The arrival times, measured with respect to the peak of the laser pulse, 
at the rear of the $32 \mu \mathrm{m}$ gold layer and at the top of the steps are shown in Figure 2. We obtained a shock velocity across the steps of $17.3+0.3 \mathrm{~km} / \mathrm{s}$. The uncertainty of $+2 \%$ is the best yet achieved in a laser-generated shock experiment and illustrates the advantages of using high laser energy, which permits the use of larger targets. The measured shock velocity corresponds to a pressure of abcut $3.5 \mathrm{TPa}$ ( $35 \mathrm{Mbar}$ ). Spectrally resolved $\mathrm{x}$-ray emission from the laser plasma was also measured in this experiment, permitting an estimate of suprathermal preheating effects to be made. We estimate that the rear of the target was heated to less than about $500^{\circ} \mathrm{C}$, whereas the brightness of the shock signal indicated shock heating of order $5 \mathrm{ev}$.

Figure 2 also shows the results of LASNEX simulations of this experiment. (7) Two-dimensional calculations (2D) were required to reproduce the experiment due to the two-dimensional character of the plasma blowoff, which reduces shock pressure. However, the shock propagation itself is planar. The two 2D-LASNEX-calculated curves are for slightly differing absorption fractions and are seen to bracket the data. The best curve is for about 30 percent total absorption.

An analysis of the LASNEX calculations which accurately simulate this experiment reveals a modified power-law scaling of peak shock pressure with intensity. (7) However, for intensities below about $3 \times 10^{15} \mathrm{w} / \mathrm{cm}^{2}, P_{S}$ scales approximately as $t_{0}^{0.6}$, with a tendency toward saturation only occuring at much higher intensity.

Figure 2. Shock arrival times measured in a thick Al-Au target (see text). The best LASNEX simulation used a total absorption of about $30 \%$. The calculated shock paths are shown as straight lines but actually curve somewhat due to shock decay.

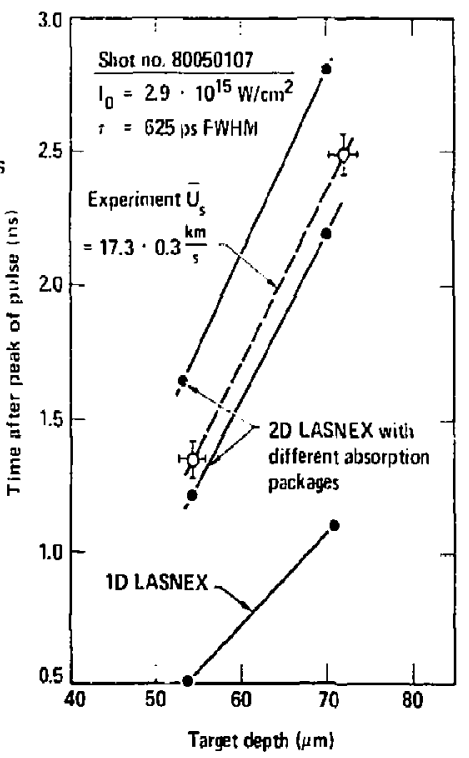

This experiment showed that preheat-free laser-generated shocks in the 3-4 TPa range are possible with present $1.06 \mathrm{jm}$ wavelength lasers. However, we know that the shock was decaying strongly as 
it entered thr step. LASNEX calculates approximately 32 percent attenuation in the pressure during the step traversal. Indeed, a peak pressure of nearly $9 \mathrm{TPa}$ deeper into target (strongly preheated, however) was predicted.

\section{Effects of Laser Wavelength}

We know of two promising methods for overcoming the problems of preheat and shock decay. The first approach $(2)$ uses a thin, dense layer imbedded in a slab target to absorb suprathermal electrons. The layer becomes very hot in this process, and its expansion after the termination of the laser pulse helps to maintain the shock pressure at a high value. LASNEX calculations $(2)$ predict that in optimally designed targets of this type the pressure does not decay substantially for several nanoseconds after the termination of the pulse (whose duration was typically $0.3 \mathrm{~ns}$ in these calculations). Note that a laser-generated shock in a homogeneous slab target is expected to decay after only about $2 \tau$, where $\tau$ is the pulse length. Tests of these predictions are presently underway in our laboratory. The principal drawback to the technique is that it is a relative inefficient use of the laser energy in that for a given intensity somewhat lower peak pressures are generated than in targets without dense layers.

The second approach is the use of laser wavelengths shorter than one micron, which has been predicted to offer considerable advantages to inertial-fusion experiments (8) and shock-wave experiments as well. These advantages include increased absorption of laser energy by the target, reduced suprathermal electrun preheat, and possible increased shock pressures. The increased absorption is a direct consequence of the dominance of inverse Bremsstrahlung scattering at short wavelength $\lambda$ and has been observed in several laboratories. (9) Reduced preheat is a consequence of the reduced importance of resonance absorption at shorter $\lambda$. Spectrally resolved $x$-ray emission measurements indicate dramatic reductions at short $\lambda$ of the number of suprathermal electrons produced and their characteristic energies. ( 9$)$

Several theoretical treatments have shown that $P_{s}$ should increase with decreasing $\lambda$. However, the scaling of $P_{S}$ with $\lambda$ is a subject of some controversy. If we write $P \propto \lambda^{-n}$, various theories predict $n=0.33-2$, indicating benefits ranging from modest to dramatic when short $\lambda$ is used. The most sophisticated of these theories $(10)$ predicts $n=1$, but only for spherical geometry; the case for planar geometry is still being studied and may differ from the case for spherical geometry.(11) In any case, the maximum pressure achievable in a preheat free region of the target should scale much more favorably with $\lambda$ than is indicated by the models, since the reduced preheat should permit measurements to be made in thinner cargets, where shock decay has not significantly attenuaced the shock.

We have recently made shock velocity measurements on aluminum targets irradiated by $0.35 \mathrm{~m}$ light produced by frequency-tripling one arm of the Argus laser. The targets (12) were aluminum slabs 
25 th thick. On the irradiated (front) side of the target, Be layers varying between 0-7 $\mathrm{km}$ thick were deposited. A 4 jm-deep, flat-bottomed channel was ion milled into the rear surface of each target. An ultrafast streak camera measured the shock arrival times at the bottom and top of the channel with 20 ps time resolution. Incicent laser intensity was nominally $1-2 \times 10^{14} \mathrm{w} / \mathrm{cm}^{2}$, with a few shots being done at higher intensity. Pulse length was typically 700 ps FWHM; absorptior. was approximately 95 percent. Arrival time data are shown in Fig. 3. These data must be regarded as preliminary in that shot-to-shot corrections for intensity variations about the nominal value have not yet been made; this should have a smoothing effect. For the shots shown in Figure 3, measured shock velocities were about $25 \mathrm{~km} / \mathrm{s}$, implying shock pressures of 1-1.2 TPa. These pressures are also inferred by the arrival time data. A simple analysis shows that for a triangular laser puise with FHHM $T$,

$$
\left(t_{a}-t\right)=\frac{L}{U_{s}}-\frac{\tau}{\left(1+\frac{2}{\alpha}\right)}
$$

where $t_{a}$ is the absolute arrival time, $t_{0}$ is the time the peak of the pulse arrives at the target, $L$ is the target thickness, $U_{S}$ is the peak value of shock velocity, and $\alpha$ is the exponent in the intensity scaling law. For $v_{s}=25 \mathrm{~km} / \mathrm{s}, \tau=700 \mathrm{ps}, \mathrm{L}=21 \mu \mathrm{m}$, and $\alpha=0.7$, Eq. (1) yields $\left(t_{a}-t_{0}\right)=300 \mathrm{ps}$, in accord with the data. The upward slope of $\left(t_{a}-t_{0}\right)$ with increasing Be thickness is due to the increased distance which the shock must traverse. A slight dip in this curve at small Be thickness is expected due to the small shock impedance mismatch between $A 1$ and Be. The above results are in good agreement with preliminary LASNEX simulations of the experiment by $\mathbf{w}$. C. Mead of LLNL.

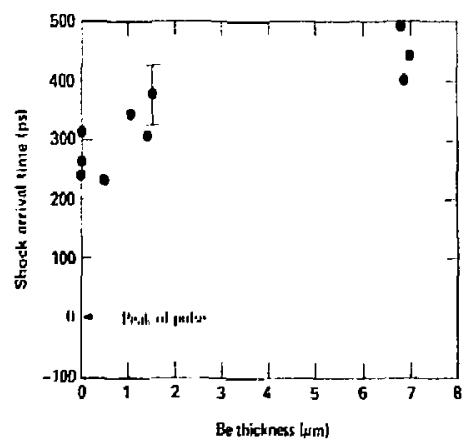

Figure 3. Shock arrival times in Be-coated aluminum targets, produced with 0.35 in wavelength irradiation and incident intensities of $1-2 \times 10^{14} \mathrm{w} / \mathrm{cm}^{2}$. 
We also irradiated one of the pure Al targets with a $1.06 \mathrm{fm}$ pulse at $3 \times 10^{14} \mathrm{w} / \mathrm{cm}^{2}$ incident intensity, keeping all other experimental parameters the same as in the 0.35 m shots. On this shot, we measured $v_{S}=20 \mathrm{~km} / \mathrm{s}$ and $\left(t_{A}-t_{0}\right)=550 \mathrm{ps}$, both corresponding to $P_{\mathrm{S}} \approx 0.6 \mathrm{TPa}$. The absorbed intensity was estimated to be about $1.2 \times 10^{14} \mathrm{w} / \mathrm{cm}^{2}$, comparable to that used in the 0.35 m experiments. Thus, we have observed a significant enhancement of $P_{S}$ by a threefold reduction in $\lambda$. We must further realize that in the 1.06 m experiment, the rear surface of the target was preheated to several thousand degrees, whereas the preheat estimated for the short $\lambda$ shots is negligibly small. In these experiments, shock decay is not believed to have been significant, since the shock wils studied at tines earlier than $2 \tau$, the approximate onset time for shock decay in $\mathrm{Al}$.

It appears, from our present vantage point, that future ultrahigh pressure shock wave experiments will be performed on lasers of high energy and short wavelength.

\section{Requirements for Physical Property Measurements}

Diverse physical property measurements have been developed for more conventional, lower-pressure experiments, where time resolution on the nanosecond scale is sufficient. Such techniques include measurement of shock and particle velocity (from which the Hugoniot is obtained), sound velocity, temperature, electrical conductivity, $x$-ray diffraction, etc. Bringing such powerful techniques to bear in laser-shock-compression experiments will require time resolution of 10 ps or better. At present, only shock velocity can be measured with such accuracy (as described above), although particle velocity and temperature measurements appear feasible.

Target requirements for laser-driven shock-wave experiments are also severe. As seen in the discussion of experiments above, targets are typically a few tens of microns thick, and shock velocity measurements are made across parallel planes typically separated by 5-15 $\mathrm{mm}$. A possible target configuration for a copper-aluminum impedance match experiment is shown in Fig. 4. Obviously, surface finishes on such targets must be of the order of $0.1 \mu \mathrm{m}$ and the interplanar distances measured to about $0.1 \mathrm{~m}$. Such tolerances are now possible.

Significant progress has been made over the past year toward fabrication and accurate characterization of targets. Reeves and Helm ${ }^{13}$ have developed a masking technique for producing smooth, uniform steps in metallic targets. A different technique has been developed by Devine and Evans, 14 using ion milling to produce high quality steps on photo-rasked metal substrates. A gold target with two $10 \mathrm{fm}$-high, ion-milled steps is shown in Fig. 5. Scanning electron raicroscopy reveals the surface roughness of these stepped surfaces to be about $0.1 \mathrm{~m}$. Several techniques have been explored for measuring the heights of such steps with high accuracy, including mechanical stylus methods, optical and scanning electron aicroscopy, and interference microscopy. The interference microscopy technique has been found superior, and the neight of a nominal 5- $\mu_{m}-h i g h$ smooth step can now be measured confidently to about $1 \%$. 


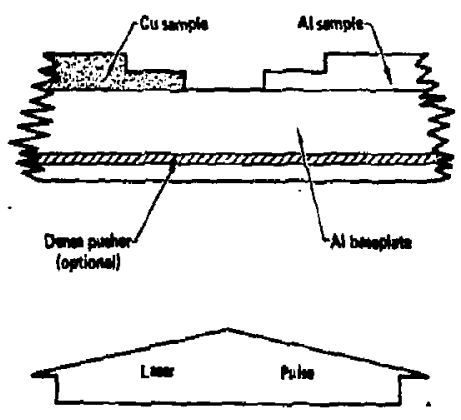

Pigure 4. Target configuration for impedance-matching experiments with laser-generated shock waves. Ster heights are typically 5-15 ja; total target thickmess is typically $20-75 \mathrm{~m}$.

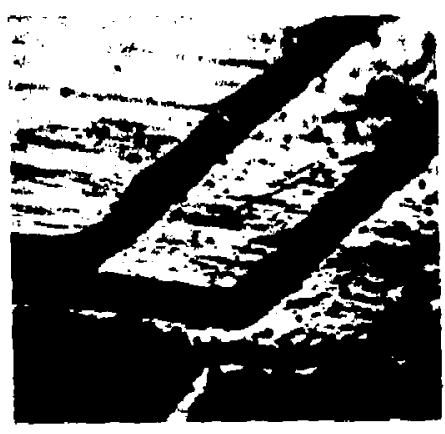

Figure 5. Sconning electron micrograph of 10 high step: ion ailled into a gold target. Sowe surface contanination due to unremoved photoresiat residue is seen.

Lecurate deternination of the initial ass density of the target is a requirewent for shockwave experiments in general and is a challeng, $\pi g$ problex for laser-driven shock experiments, where the total target mass an be only a few ng. Recently, Veeser and Maggiore $(15)$ have developed a technique using low-energy proton backscatter to measure areal density in very small specimens. The technique may provide initial density measurenents approaching $1 \%$ accuracy. In addition, the wethod hes high spatial resolution $(<50 \mathrm{\omega})$, so it can detect denuity variations in different steps.

Recently, we began a series of shock impedance-atching experiants, using the Janus laser to irradiate copper-aluainu targets. The details of the experiment are discussed elsewhere in these proceedings.(16) Prelivinary experimental results are shom in Fig. 6, and are compared to both the present capability to do such experineats uoing other laboratory athods sad to our projected capabilities using upsraded laser sources and realistic disgostic improvewents.

Very accurate data are poacible using two-atage, light-gas guns, vith sock velocity uncegtainities dear $1 \%$, ug to pressures of about $0.15 \mathrm{TR}$ in eluaine. (27) Present Jemus experimats just overlap the two-utage gui range ad extend up to pressures of shout 0.6 TPa in alunimen. The overlep, though limited, obould be useful since it vill sllow an accurate eleck to be whe of the lowpresente

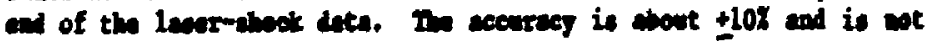


sufficient to test competing EOS theories. The relatively large scatter is due primarily to laser intensity variations produced by insufficient flatness of the targets, the use of fast irradiating optics, and degraded space/time resolution of the imaging system. (16) These sources of scatter are being eliminated for upcoming experiments, in which we expect the data accuracy to improve to about $+2 \%$. An upgrading of Janus should also extend the range of the data to about $1.2 \mathrm{TPa}$. It should be mentioned here that in all experiments conducted so far at Janus, the desire to study preheat-free shocks has necessitated target thickness so large that shock decay is inevitable. As mentioned above, the use of imbedded pushers and/or shorter laser wavelengths offer solutions to this dilema. When Novette is complete in late 1982, the capability will exist to perform high-energy irradiations at stort wavelengths $(0.53$ or $0.35 \mu \mathrm{m})$, and accurate shock impedance data up to the $4 \mathrm{TPa}$ range and perhaps beyond, will become possible, thus entering the repime where similar experiments are presently being performed with anclear-explosives drivers. (18)

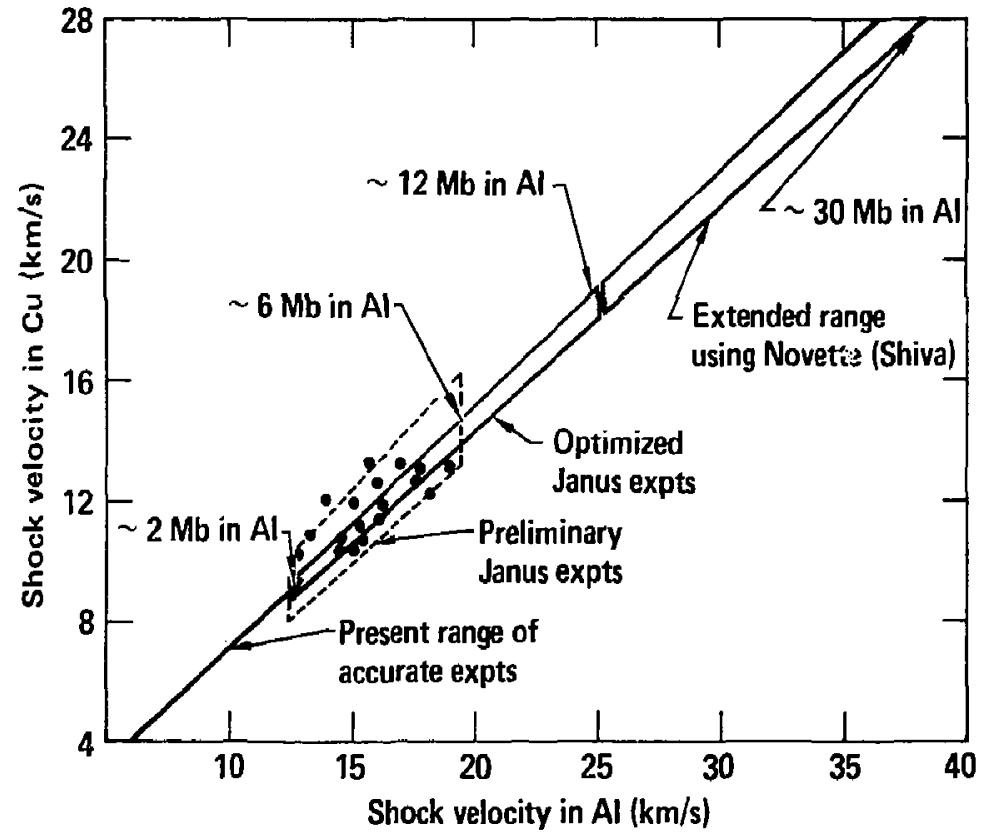

Figure 6. Preliminary results of Al-Cu impedance matching experiments. Dashed lines represent $10 \%$ error limits in data. Solid line at pressure below $0.2 \mathrm{TPa}(2 \mathrm{Mb})$ in $\mathrm{Al}$ is the present range of accurate experiments. Optimized experiments at Janus (see text) should extend to about $1.2 \mathrm{TPa}$. Novette could produce data at pressures beyond $3-4 \mathrm{TPa}$. 


\section{Acknowledgments}

The work described above involved many cullaburaturs. The Shiva shock velocity measurements were performed in collaboration with D. Banner and D. Phillion. The short wavelength experiments were done in collaboration with E. M. Campbell, W. C. Mead, R. E. Turner, and $\mathrm{F}$. Ze. The impedance matching experiments vere performed jointly with 2 . Veeser of Los Alamos National haboratory. $Y$. $T$. Lee and R. J. Olness collaborated in the numerical simulations of several of these experiments. We also thank the crews of the Janus, Argus, and Shiva laser facilities for their skillful operation. We also acknowledge W. Hatchet, G. Devine, L. Evans, and $G$. Reeves (LANL) for fabricating the high quality targets used in these experiments.

\section{References}

1. R. E. Kidder, Nucl. Fusion 8, 3 (1968).

2. R. M. More, in Laser Interaction and Related Plasma Phenomena, Vo1. 5, edited by H. J. Schwarz and H. Hora, Plenum, 1981, p. 253 .

3. G. B, Zimmerman and พ. L. Kruet, Comments Plasma Phys. 2, 51 (1975).

4. J. Grun, R. Decoste, B. M. Ripin, and J. Gardner, NRL Memorandum Rept. 4410, 1981.

5. L. Veeser and J. Solem, Phys. Rev. Lett. 40, 1391 (1978); R. J. Trainor, J. W. Shaner, J. M. Auerbach, N. C. Holmes, Phys. Rev. Lett. 42, 1154 (1979).

6. M. H. Key, Nature 283,715 (1980).

7. R. H. Harrach, Y. T. Lee, R. J. Trainor, N. C. Holmes, M. D. Rosen, D. L. Banner, R. J. Olness, Shock Waves in Condensed Matter, AIP Conf. Proc. (these proceedings).

8. C. Max, Laser Program Annual Report, UCRL-50021-78, P. 3-56 (1979).

9. E. Fabre, et al., 21st Annual Meeting of Div. of Plasma Physics, American Physical Society, 1979; F. Ze, et al., Laser Program Annual Report - 1979, UCRL 50021-79, p. 6-46 (ī980).

10. C. Max, C. G. McKee, H. C. Mead, Phys. Rev. Lett. 45, 18 (1980).

11. C. Max, R. Fabbro, and E. Fabre, Bull. Am. Phys. Soc., 25, 895 (1980).

12. W. Hatcher and G. Devine of LLML were responsible for target fabrication.

13. G. A. Reeves, P. J. Helm, CLEOS/ICF 80, Feb, 26-28, 1980, San Diego, Calif.

14. G. Devine and L. Evans, (unpublished).

15. L. Veeser and C. Maggiore, (to be published).

16. N. C. Holmes, R. J. Trainor, R. A. Anderson, L. R. Veeser, and G. A. Reeves, Shock Waves in Condensed Matter, AIP Conf. Proc. (these proceedings).

17. A. C. Mitchell and W. J. Nellis, J. Appl. Physics 52, 3363 (1981).

18. C. E. Ragan, B. C. Diven, M. Rich, W. A. Teasdale, Shock Waves in Condensed Matter, APS Conf. Proc. (these proceedings); C. E. Ragar. Ií, Phys. Rev. A 21, 458 (1980). 